\title{
The Design of Calibration Equipment for Dynamic Loading of Optoelectronic Six-Component Force-Torque Sensor
}

\author{
Milan Kvasnica \\ Tomas Bata University in Zlin, Faculty of Applied Informatics \\ Nad Stráněmi 4511, CZ-76005 Zlín, Czech Republic \\ E-mail: kvasnica@fai.utb.cz
}

\begin{abstract}
The paper deals with current state of the art in technology in the field of the sixcomponent sensory system for the measurement of axial shiftings and angular displacements with application for the force-torque measurement, haptic interaction and their principles of activity. The paper is focused on the design of universal calibration equipment for the sixcomponent force-torque sensors allowing the measurements of their parameters while the multi-component dynamic loading. This paper is focused on the design of the calibration equipment for the six-component force-torque sensors. There are introduced for the comparison two different types of calibration equipments. The first, known variant is destined for the calibration at static loading. The second, new variant, using the one-axis resistance strain gauges is destined for the measurements of the force-torque sensors and their components at both static and dynamic loading. The dynamic loading force is acting by the use of the pressing rods moved from the mechatronics, hydraulic or pneumatic actuators against the basic points of the squirrel cage of the calibration equipment in order to enable the measurement not only the static, but as well the dynamic characteristics of six-component sensor for more components simultaneously.
\end{abstract}

\section{INTRODUCTION}

The paper deals with current state of the art in technology in the field of the sixcomponent sensory systems for the measurement of axial shiftings and angular displacements focused on problems connected with the design and calibration of six-component force-torque sensory systems. Described modular sensory system enables the six Degrees of Freedom sampling of micro-displacements for vibration measurement as well as the macrodisplacements at static and dynamic loading of engineering constructions. The modularity of described six DoF sensory systems enables the tailoring of properties for the applications in robotics, human-machine interface and in assistive technologies such as:
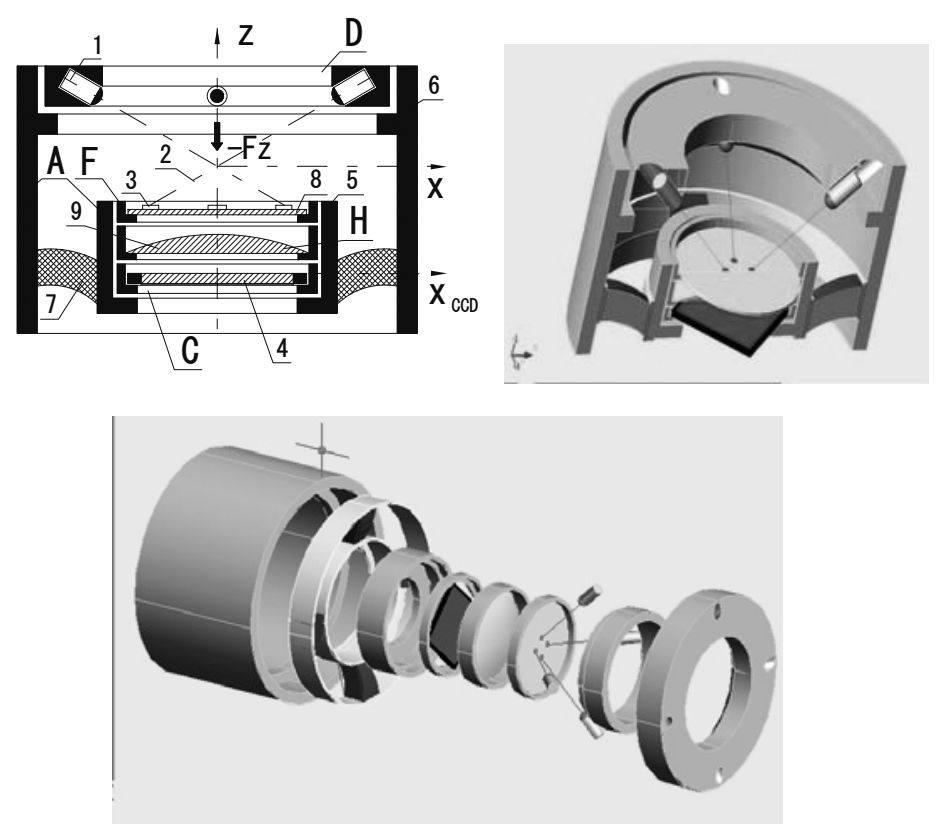

Figure 1: Six-component force-torque transducer (from UTB Zlin) 
Portable modular system for the six-component static and dynamic measurement in general anisotropic construction in 3-D space; Six-component detection of micro-elastic or macroelastic deformation; Six-component force-torque sensors of various properties; Tactile sensors; Six-component joystick; Accelerometers; Dynamic weighing; Active compliant links; On-line checking of the dynamic activity of vehicles; Human-machine interface with the haptic interaction; Signature scanners for banking; Keyboards for blind people; Chaser systems for antiterrorist robots; Artificial limbs.

The substance of described sensory system is based on the shape of the 3D pyramid consisting of four edges created by light rays (respectively. walls of light planes from structured light). The intersection of the laser light rays is forming the apex of the pyramid. The (square) basis of the pyramid is created by means of the 2-D CCD (Charge Coupled Device) (or the PSD - Position Sensitive Device, or CMOS) array and is used like a floating coordinate frame $x, y$. Intersection of four light rays with the 2-D CCD array in the basic parallel position between both two flanges results in a square shape with four light spots in the corners. Intersection of four light rays against the 2-D CCD array under acting of axial shiftings and angular displacements generally results in a trapezoidal position of the light spots. The light rays pyramid is coupled with the outer flange and the 2-D CCD array is coupled with the inner flange. The six DoF motion (three axial shiftings and three angular displacements) between two flanges is sampled by means of the unambiguous trapezoidal light spots position.

Simple modular construction enables low cost customization, according to the demanded properties: A -stiff module of two flanges connected by means of microelastic deformable medium; B -compliant module of two flanges connected by means of macroelastic deformable medium; C -the module of the 2-D CCD array; D -the module of insertion flange with basic light sources configuration and focusing optics; $\mathrm{F}$-the module of the plane-focusing screen; $\mathrm{H}$ the module of the optical member for the magnifying or reduction of the light spots configuration. The problem of the customization of six-DoF sensory systems according to the enhanced accuracy and operating frequency of scanning of the six-DoF information is possible to improve by means of the module of insertion flange with the configuration of light sources with strip diaphragms, creating the light planes with strip light spots and by means of the module of the single or segmented linear or annular CCD or PSD elements with higher operating frequency, respectively using the module of two, parallel working, concentric CCD annulars with higher reliability.

The explanation of the activity of the force-torque transducer: Laser diodes 1 emit the light rays 2 creating the edges of a pyramid intersecting the plane of the 2-D CCD array, here alternatively the focusing screen 8 with light spots 3 , see Figure 1 . The unique light spots configuration changes under axial shifting and angular displacements between the inner flange 5 and the outer flange 6 connected by means of elastic deformable medium 7. An alternatively inserted optical member 9 (for the magnification of micro-movement, or the reduction of macro-movement) projects the light spots configuration from the focusing screen onto the 2-D CCD array 4. Four light rays simplify and enhance the accuracy of the algorithms for the evaluation of the six-DOF information.

The algorithms for the computation of three axial shiftings and three radial displacements values is based on the inverse transformation of the final trapezoidal position of four light spots related to the original square light spots position in the plane coordinate system $X_{C C D}$, $y_{C C D}$ on the 2-D CCD array. This algorithm determines the relative location and orientation of a floating 2-D coordinate system against a fixed 3-D coordinate system corresponding to the apex of the pyramid shape, or contrary. The information about three axial shiftings and three angular displacements is sampled and converted according to a calibration matrix to acting forces $\mathrm{Fx}, \mathrm{Fy}, \mathrm{Fz}$ and torques $\mathrm{Mx}, \mathrm{My}, \mathrm{Mz}$, creating the vector $\mathrm{F}=[\mathrm{Fx}, \mathrm{Fy}, \mathrm{Fz}, \mathrm{Mx}, \mathrm{My}, \mathrm{Mz}]^{\top}$. The vector $D=K$.F of axial shiftings and angular displacements on the output of the six-component sensor enables the evaluation of the vector $F=K^{(-1)} . D$, where the $K$ is the characteristic matrix and the $\mathrm{K}^{(-1)}$ is the compensation matrix. The calibration matrix describes the properties of the elastic deformable medium in the force-torque sensor. The aim of the calibration equipment is to determine the interference of every force-torque component to remaining components and to determine the linear part of the working characteristic. 


\section{THE DESIGN OF THE CALIBRATION EQUIPMENT FOR STATIC LOADING OF THE SIX- COMPONENT FORCE-TORQUE SENSORY SYSTEM}

For the calibration of the six-component force-torque sensory systems are used two different types of calibration equipments, for the static loading and for the dynamic loading. The first, known variant is destined for static calibration of force-torque sensors according to design of the STU Bratislava. This calibration equipment consists of six independent loading corridors for every force-torque component separately, see Figure 2. Here the acting forces Fx, $\mathrm{Fy}, \mathrm{Fz}$ and torques $\mathrm{Mx}, \mathrm{My}, \mathrm{Mz}$, create the vector $\mathrm{F}=[\mathrm{Fx}, \mathrm{Fy}, \mathrm{Fz}, \mathrm{Mx}, \mathrm{My}, \mathrm{Mz}]^{\top}$.

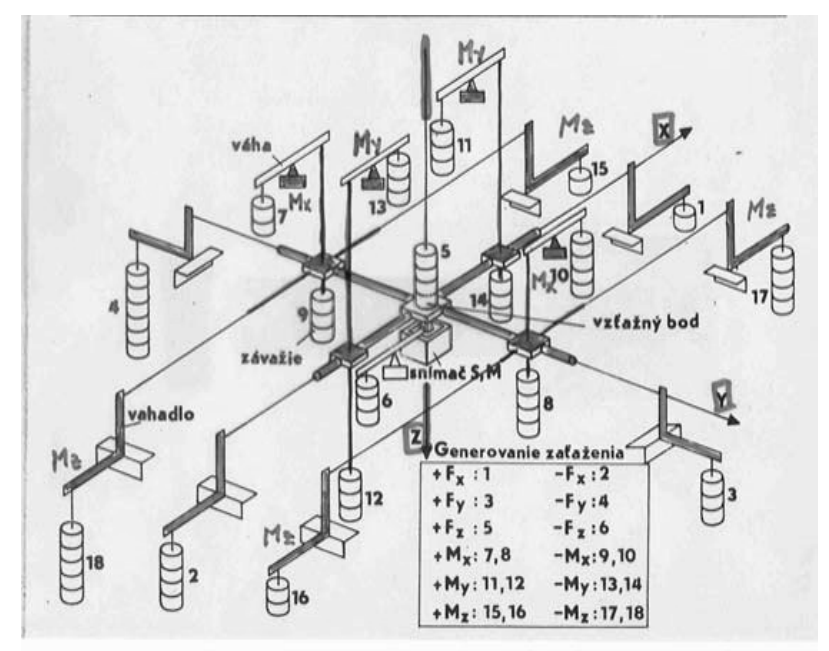

Figure 2: Calibration equipment for static loading (from STU Bratislava)

The first variant of the calibration equipment destined for the static calibration which is sufficient mostly for force-torque sensory system with longer response time. Some elastic deformable medium in the force-torque sensory system are suitable only for the sampling of force-torque process with low frequency, because some materials of the elastic deformable medium interfere significant to remaining components.

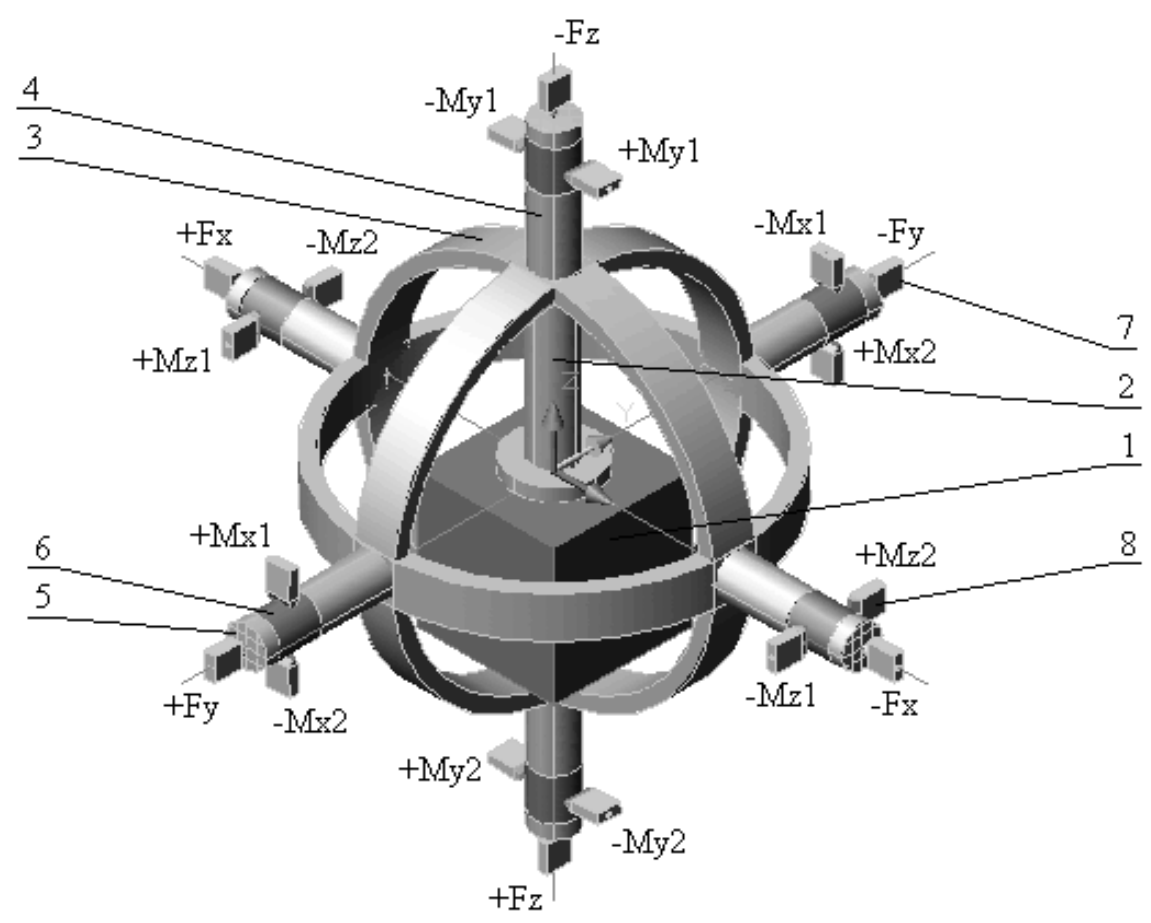

Figure 3: Calibration equipment for dynamic loading (from UTB Zlin) 


\section{THE DESIGN OF THE CALIBRATION EQUIPMENT FOR DYNAMIC LOADING OF THE SIX- COMPONENT FORCE-TORQUE SENSORY SYSTEM}

Following is described the second new variant of the calibration equipment destined for the sampling of the six-component force-torque sensors at dynamic loading from the UTB Zlin. In this calibration equipment are used the one-axis resistance strain gauges for the measurements of the force-torque components at both static and dynamic loading. The dynamic force-torque loading is acting by the use of the pressing rods moved from the mechatronic, hydraulic or pneumatic actuators against the basic points of the squirrel cage of the calibration equipment. This enable the measurement not only the static, but as well the dynamic characteristics of the six-component force-torque sensor for more components simultaneously.

In Figure 3 is depicted the design of mechanical parts of the squirrel cage between actuators and strain gauges: 1. Six-component sensor; 2. Axial lever arm; 3. Stiff cradle; 4. Grapple; 5 . Contact surface of the force-torque acting; 6 . Contact surface for the axial $x, y, z$ torque acting; 7 . One-axis strain gauges unit for the axial $x, y, z$ force acting; 8 . Strain gauges units for the angular torque acting.

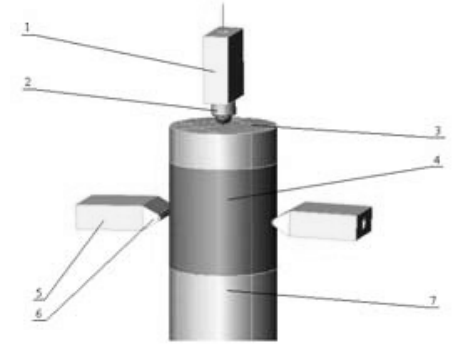

Figure 4: The emplacement of tensometric dynamometers on the arms

In Figure 4 is depicted the design of the emplacement of tensometric dynamometers on the arms: 1 . One axis tensometric dynamometer; 2 . The finger for the force acting in axes $x, y, z ; 3$. The bearing of the finger destined for the acting in axes $x, y, z ; 4$. The needle shaped rolling bearing creating the seating face of fingers causing the torque acting with rotation axis $x, y, z ; 5$. One axis tensometric dynamometer; 6 . The finger for the torque acting with rotation axis $x, y, z ; 7$ Axial lever arm.

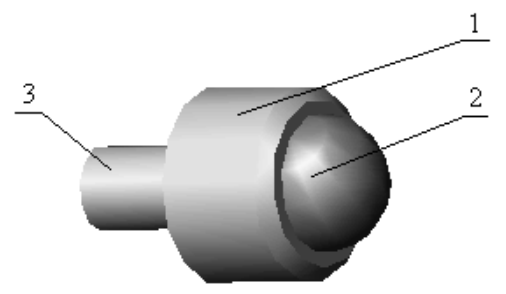

Figure 5: The finger of the force acting in axes $x, y, z$

In Figure 5 is depicted the design of the force acting in axes $x, y, z: 1$. The body of the finger; 2 . The rolling bullet; 3 . The winding.

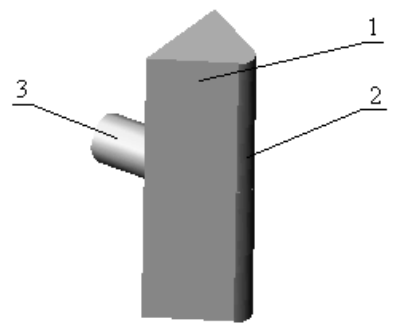

Figure 6: The finger of the force transfer causing the torque 
In Figure 6 is depicted the design of the force transfer causing the torque: 1 . The body of the finger; 2 . Rotatively beared needle; 3 . The winding.

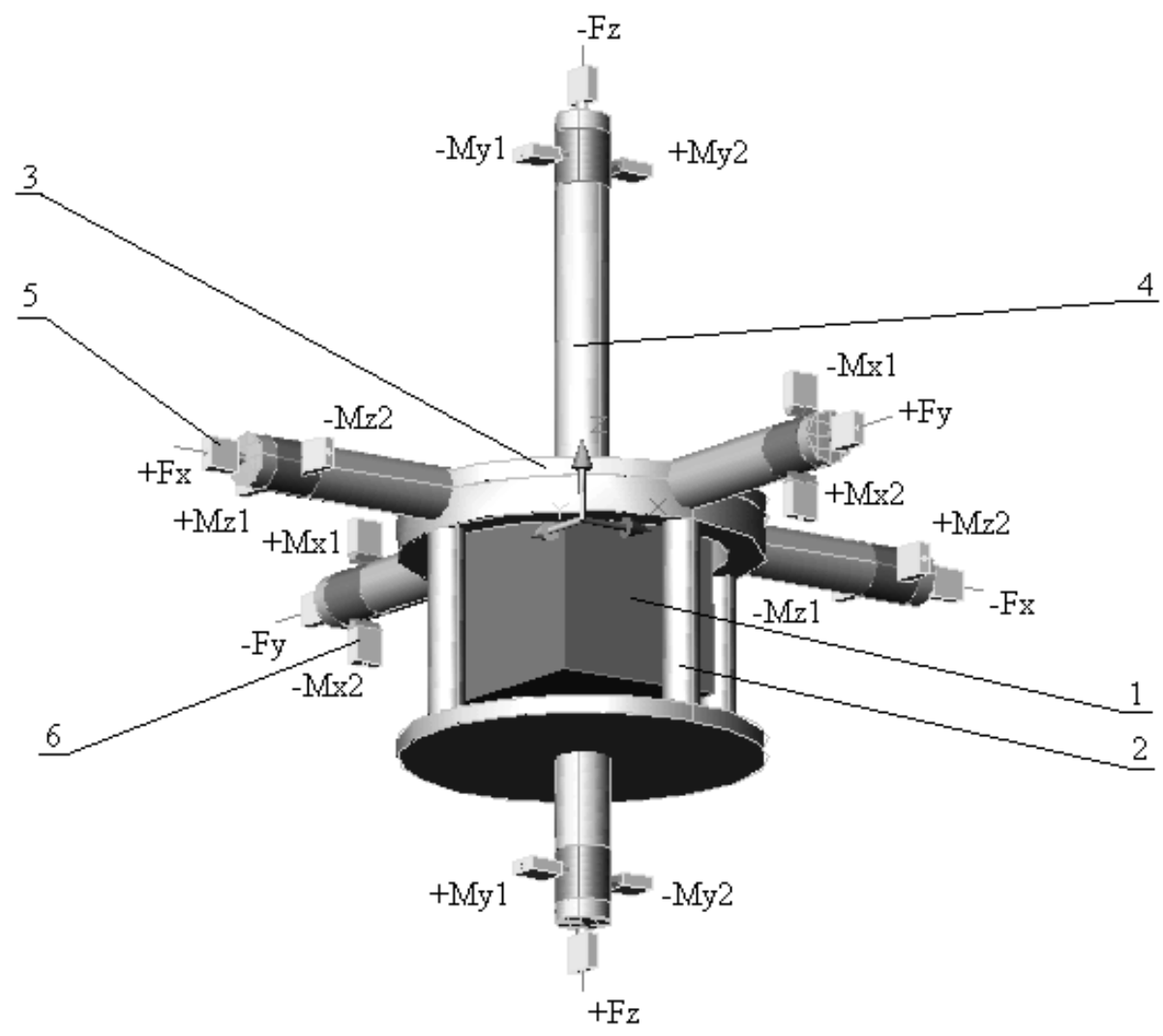

Figure 7: The design of the grapple

In Figure 7 is depicted the design of the grapple: 1. Examined six-component forcetorque sensor; 2 . Firm part of the stiff cradle; 3 . Detachable part of the stiff cradle; 4 . The arm with rolling bullet and rotatively beared needles; 5 . One axis tensometric dynamometer with rolling bullet; 6. One axis tensometric dynamometer with rotatively beared needles.

\section{CONCLUSION}

The design of universal calibration equipment for the six-component force-torque sensors is focused on simultaneous measurements of force-torque parameters for six degrees of freedom while the multi-component dynamic loading in order to determine the behavior of elastic deformable medium.

The modular design of six component sensory system for sampling of three axial shiftings and three angular displacements presented here enables easy customizing for a wide variety of applications. Various combinations of the modular components enable tailoring of the sensory system properties including the use of the haptic interface for applications such as: detection of microelastic or macroelastic deformation, active compliant links, multi-DoF hand controllers, signature scanners, keyboards for blind people, tactile sensors, and range finders-positioners. In general, this modular design concept allows:

- Maximization of service life because of ease of repair and the use of modular components for various types of sensors.

- Customization for a wide variety of design requirements. For example: various levels of resolution and operating frequency, enhanced demands for safety and reliability in space robotics and medical use, and low cost design for manufacturing.

In conclusion, many fields could benefit from the use of such a sensory system: robotics, telerobotics, and measurements in engineering constructions, intelligent automation, automotive and aerospace industry, medicine, defense and banking. 


\section{ACKNOWLEDGEMENT}

The support from the grant Vyzkumne zamery MSM 7088352102 „Modelování a rizení zpracovatelských procesů prírodních a syntetických polymerů" RP370460007 is gratefully acknowledged.

\section{REFERENCES}

[1] Hirzinger G., Dietrich J., Gombert J., Heindl J.,Landzettel K.,Schott J.: "The Sensory and Telerobotic Aspects of Space Robot Technology Experiment ROTEX", Proceedings of the International Symposium on Artificial Intelligence, Robotics and Automation in Space, 1992, Toulouse, Labege, France.

[2] Kvasnica M.: "Six-Component Force-Torque Sensing by Means of One Quadrate CCD or PSD Element", Proceedings of the 2nd International Symposium on Measurement and Control in Robotics, 1992, AIST Tsukuba Science City, Japan.

[3] Kvasnica M.: „Intelligent Sensors for the Control of Autonomous Vehicles”, Proceedings of the 6th International Conference and Exposition on Engineering, Construction and Operation in Space and on Robotics for Challenging Environments - Space and Robotics 98, Albuquerque, New Mexico, April 1998, USA.

[4] Kvasnica M.: "Measurement in Engineering Construction and Control Operations in Space”, ASCE Multi-Conference on Engineering, Construction, Operations, and Business in Space and on Robotics for Challenging Situations and Environment, Space and Robotics'2000, Albuquerque, New Mexico, USA.

[5] Kvasnica M.: „Improvement of Positioning Accuracy in Multi-Pod Parallel Structures”, ASCE Multi-Conference on Engineering, Construction, Operations, and Business in Space and on Robotics for Challenging Situations and Environment "Space and Robotics 2002", Albuquerque, New Mexico, February-March 2002, USA.

[6] Kvasnica M.: "Modular Force-Torque Transducers for Rehabilitation Robotics” Proceedings of the 6th International Conference on Rehabilitation Robotics ICORR'1999, Stanford University, July 1999, Stanford, USA.

[7] Kvasnica M., "The Design of the Calibration Equipment for the Six Component Force-Torque Sensor". Proceedings of the International Congress Mechatronics and Robotics MiR 2007, Izdatelstvo poligraficeskij komplex Lenexpo 2007, Saint Petersburg, Russia.

[8] Kvasnica M. : "Six DoF sensory system for the force-torque control of walking humanoid”. Proceedings of the 11th International Conference on Climbing and Walking Robots and the Support Technologies for Mobile Machines, CLAWAR'2008, Coimbra, Portugal.

[9] Palka M. : "The Design of the Calibration of the Six-Component Force-Torque Sensor", Diploma Thesis, Tomas Bata University in Zlin, Czech Republic, 2007.

[10] Sásik J. : "Multi-component Force-Torque Sensors Calibration Methods for Robotics Application", Strojnicky casopis, 38, No.6, 1987, Bratislava, Slovakia. 